\title{
Episodic Vestibulocerebellar Ataxia Associated with a CACNA1G Missense Variant
}

\author{
José Gazulla ${ }^{a}$ Silvia Izquierdo-Alvarez ${ }^{b} \quad$ Emilio Ruiz-Fernández $^{\text {a }}$ \\ Alba Lázaro-Romero ${ }^{a}$ José Berciano ${ }^{c}$ \\ aDepartment of Neurology, Hospital Universitario Miguel Servet, Zaragoza, Spain; bSection \\ of Genetics, Department of Clinical Biochemistry, Hospital Universitario Miguel Servet, \\ Zaragoza, Spain; 'Department of Neurology, Hospital Universitario Marqués de Valdecilla \\ (IDIVAL), University of Cantabria, CIBERNED, Santander, Spain
}

\section{Keywords}

Vestibular hypofunction - CACNA1G - Episodic ataxia type 10 - Autosomal dominant . Carbamazepine - Episodic vestibulocerebellar ataxia type $10 \cdot$ Episodic ataxia

\begin{abstract}
Episodic vestibulocerebellar ataxias are rare diseases, frequently linked to mutations in different ion channels. Our objective in this work was to describe a kindred with episodic vestibular dysfunction and ataxia, associated with a novel CACNA1G variant. Two individuals from successive generations developed episodes of transient dizziness, gait unsteadiness, a sensation of fall triggered by head movements, headache, and cheek numbness. These were suppressed by carbamazepine (CBZ) administration in the proband, although acetazolamide and topiramate worsened instability, and amitriptyline and flunarizine did not prevent headache spells. On examination, the horizontal head impulse test (HIT) yielded saccadic responses bilaterally and was accompanied by cerebellar signs. Two additional family members were asymptomatic, with normal neurological examinations. Reduced vestibulo-ocular reflex gain values, overt and covert saccades were shown by video-assisted HIT in affected subjects. Hearing acuity was normal. Whole-exome sequencing demonstrated the heterozygous CACNA1G missense variant c.6958G>T (p.Gly2320Cys) in symptomatic individuals. It was absent in 1 unaffected member (not tested in the other asymptomatic individual) and should be considered likely pathogenic. CACNA1G encodes for the pore-forming, $\alpha 1 G$ subunit of the T-type voltage-gated calcium channel (VGCC), in which currents are transient owing to fast inactivation, and tiny, due to small conductance. Mutations in CACNA1G cause generalized absence epilepsy and adult-onset, dominantly inherited, spinocerebellar ataxia type 42 . In this kindred, the aforementioned CACNATG variant segregated with disease, which was consistent with episodic vestibulocerebellar ataxia. CBZ proved successful in bout prevention and provided
\end{abstract}


symptomatic benefit in the proband, probably as a result of interaction of this drug with VGCC. Further studies are needed to fully determine the vestibular and neurological manifestations of this form of episodic vestibulocerebellar ataxia. This novel disease variant could be designated episodic vestibulocerebellar ataxia type 10 .

(C) 2021 The Author(s).

Published by S. Karger AG, Basel

\section{Introduction}

Episodic ataxias (EAs) are conditions characterized by spells of cerebellar incoordination, sometimes associated with progressive ataxia. The aim of this work was to detail the clinical characteristics of ataxic episodes in a kindred with c.6958G $>$ T (p.Gly2320Cys), a variant in the CACNA1G gene.

\section{Methods and Patients}

The pedigree of the family under study (Fig. 1) comprised 4 affected individuals over 3 generations, who displayed a similar pattern of episodic instability. A clinical description of the 2 surviving subjects (IV-2 and III-2) is provided below. Two unaffected additional family members (IV-1 and III-1) are described to assess disease segregation. Informed consent was

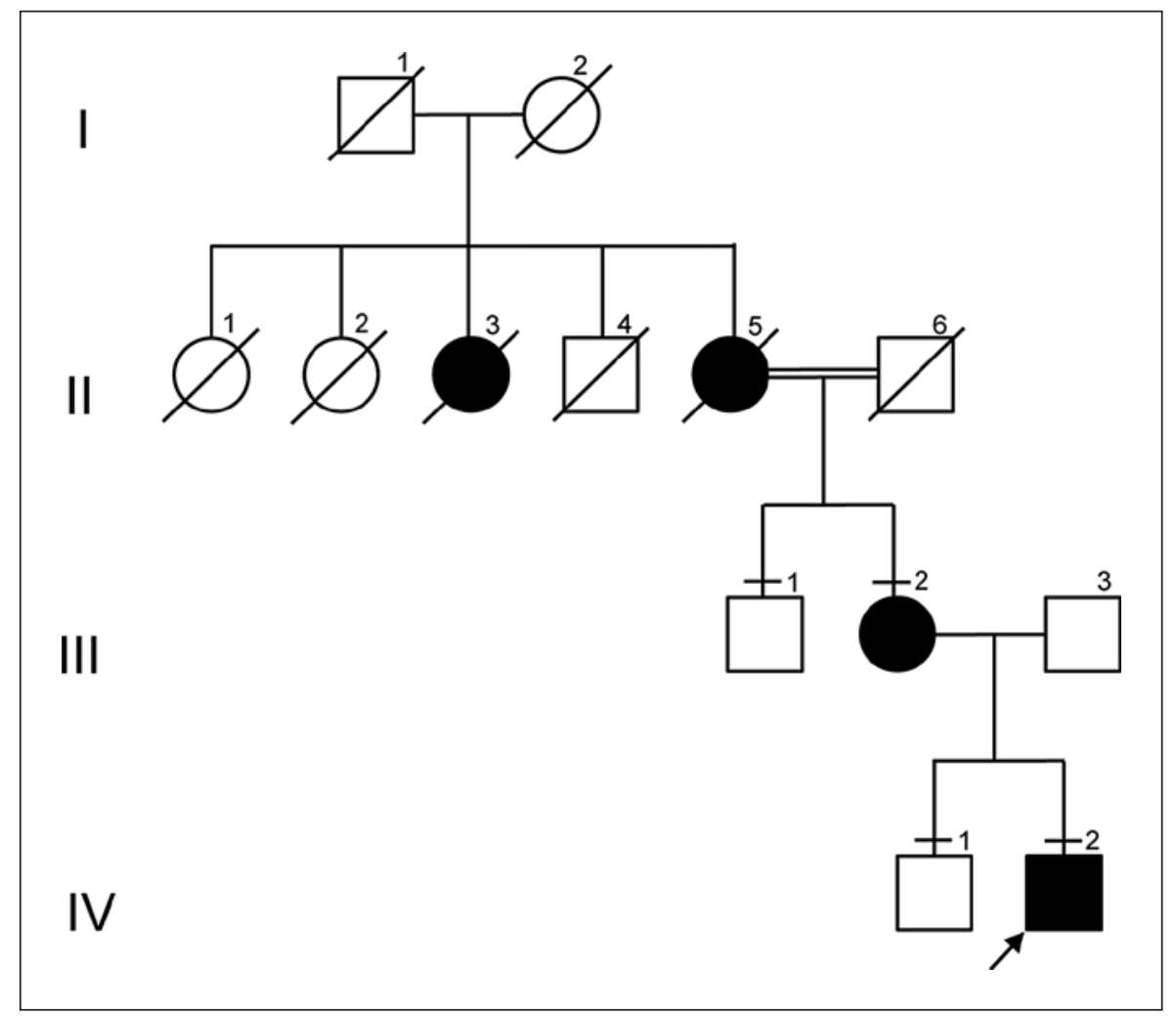

Fig. 1. Pedigree of the family. Horizontal bars under numbers indicate examined subjects; arrow indicates proband; slash lines indicate deceased individual. Squares represent males, and circles, females. Filled symbols indicate affected subjects, and empty symbols, unaffected subjects. 
obtained for every test performed and for publication. This study was made in accordance with the local institutional Ethics Committee and the 1964 Declaration of Helsinki.

Case IV-2 (Proband)

A 45-year-old man started experiencing bouts of dizziness and gait unsteadiness, aged 28. The episodes consisted of dizziness, and unstable stance and gait, which appeared spontaneously or were triggered by sudden head turns, anxiety, physical exhaustion, stress at work, and lack of sleep. The episodes lasted between $3 \mathrm{~min}$ and $2 \mathrm{~h}$, occurred weekly, and left residual instability during $24 \mathrm{~h}$. He was asymptomatic between attacks.

On 3 occasions, he reported more severe and prolonged episodes that started with an oppressive right frontoparietal headache, accompanied by right cheek numbness, unstable stance and gait that worsened in the dark, cognitive slowing, dysarthria, and a feeling of ear fullness. These spells lasted from a few weeks to 4 months, with fluctuations in intensity lasting hours or days. The patient was also aware of a sensation of fall triggered by head movements, as well as of truncal sway while sitting. Rotatory vertigo, hearing loss, tinnitus, or hypoacusis were not noted.

Dizziness and instability worsened while receiving acetazolamide, $500 \mathrm{mg} /$ day during 8 days, and topiramate, $100 \mathrm{mg} /$ day for 4 weeks, and the headache did not improve with oral sumatriptan $50 \mathrm{mg}$, nor was prevented by amitriptyline, $25 \mathrm{mg} /$ day for 6 weeks, or flunarizine, $10 \mathrm{mg} /$ day during 8 weeks. Each severe episode was improved by empiric treatment with carbamazepine (CBZ), $200 \mathrm{mg}$ twice a day. During the last 8 months, CBZ initially decreased the severity of the milder spells and prolonged the interval between them, finally suppressing them during the last 6 months.

On ictal examination, horizontal head impulse testing (HIT) yielded a bilateral saccadic response. Weber and Rinne tests were normal. Upper-limb tendon reflexes were brisk, with knee and ankle clonus, and plantar responses were flexor. Truncal sway was present during stance, and the Romberg test was positive. Gait was wide-based, and tandem gait was not possible. There was dysmetria in the heel-to-shin manoeuvre. Eye movements were full and ocular pursuit was smooth, without nystagmus.

\section{Case IV-1}

The asymptomatic 49-year-old brother of the proband had normal vestibular and neurological examinations.

\section{Case III-2}

The proband's 72-year-old mother described bouts of dizziness, gait unsteadiness, visual blurring, mental slowing and fatigue, since the age of 45 . The episodes lasted from 30 to 60 min, and up to $24 \mathrm{~h}$ on severe occasions. The patient reported that they were triggered by weather changes bringing windy spells in winter and humid heat in summer; symptoms worsened with neck flexion and while adopting orthostatism, and took place twice weekly.

On examination, horizontal HIT was saccadic bilaterally, and Weber and Rinne tests were normal. Lower-limb tendon reflexes were brisk, and plantar responses were flexor. There was truncal sway during stance, with a positive Romberg test. Gait was slightly wide-based, and tandem gait was limited to 3 steps. Mild distal tremor was present in the finger-to-nose manoeuvre, and slight dysmetria, in the heel-to-shin manoeuvre. Eye movements were full, without nystagmus.

Case III-1

The asymptomatic 77-year-old brother of patient III-2 had a normal HIT response and neurological examination. He declined further clinical and genetic testing.

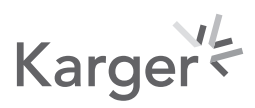




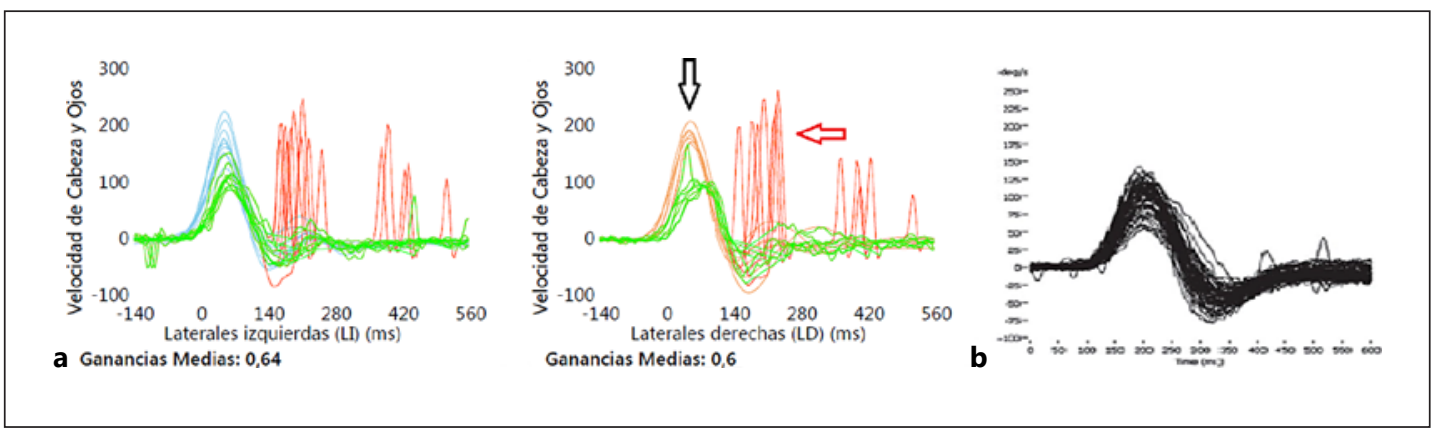

Fig. 2. a Video-assisted head impulse test in case III-2, demonstrating reduced gain values in both horizontal semicircular canals, together with overt (red arrow) and covert (black arrow) saccades. b Normal videoassisted head impulse test.

\section{Results}

Case IV-2

A video-assisted HIT (v-HIT) showed reduced vestibulo-ocular reflex (VOR) gain values in the planes of horizontal, anterior, and posterior semicircular canals bilaterally, together with overt and covert saccades. Bilateral bithermic caloric testing yielded severely reduced responses on both sides. No nystagmus was recorded on a videooculogram. Normal hearing was demonstrated by bone and air conduction audiometry.

Auditory-evoked potentials were normal. A cranial MRI disclosed no abnormalities; protein, glucose, and cell count in cerebrospinal fluid were normal; motor and sensory conduction velocities measured in the median, ulnar, and peroneal nerves, as well as somatosensory evoked potentials, were also normal.

Whole-exome sequencing, performed using SureSelectXT libraries sequenced into a Novaseq 6000 System (Illumina), revealed the exon 38 CACNA1G variant c.6958G>T (p.Gly2320Cys), in heterozygosis. This variant presented an autosomal dominant pattern of inheritance, its allelic frequency being $48 \%$. The allelic frequency was verified through the public database gnomAD, being $0.003 \%$, and was representative of the Spanish population.

It is a missense variant, in which in silico prediction algorithms were found to be $3 / 8$. The pathogenicity of this variant was estimated using 8 prediction systems included in the ANNOVAR package (SIFT, PolyPhen2, MutationTaster, MutationAssessor, LRT, FATHMM, MetaSVM, and CONDEL).

Next-generation sequencing of EA genes KCNA1 and CACNA1A, and of familial hemiplegic migraine genes CACNA1A, ATP1A2, PRRT2, and SCN1A, was negative. A molecular study performed with Triplet-repeated Primed Polymerase Chain Reaction of spinocerebellar ataxia (SCA) type 6, was also negative.

\section{Case IV-1}

This individual tested negative for the aforementioned CACNA1G variant (case III-2).

A v-HIT demonstrated reduced VOR gain values in the plane of each semicircular canal, and overt and covert saccades (Fig. 2). Normal hearing was demonstrated by bone and air conduction audiometry. The patient rejected vestibular caloric testing. Sanger sequencing demonstrated the CACNA1G variant c.6958G>T (p.Gly2320Cys) in heterozygosis. 


\section{Discussion}

EAs are dominantly inherited, genetically heterogeneous diseases, in which clinical variability is common [1,2]. Nine forms of EA have been described to date, including the recently incorporated UBR4- and FGF14-related EAs [3, 4]. KCNA2- and SCN2A-associated EAs seem to be additional forms of disease [5, 6], apart from multiple-isolated cases. Vestibular manifestations have been identified in 2 EA types (EA3 and EA4) [7, 8].

EAs are often caused by mutations in ion channel genes as KCNA1 (EA1), CACNA1A (EA2), KCNA2 and SCN2A, or in their auxiliary subunit genes, such as CACNB4 (EA5). CACNA1G encodes for the pore-forming, $\alpha 1 \mathrm{G}$ subunit of the T-type ( $\mathrm{T}$, because currents are transient owing to fast inactivation, and tiny, due to small conductance) voltage-gated calcium channel (VGCC), or $\mathrm{Ca}_{\mathrm{v}} 3.1$. The $\alpha 1 \mathrm{G}$ subunit is responsible for voltage sensing, ion selectivity and conduction, binding of channel blockers, and activation/inactivation kinetics. T-type VGCCs are low-voltage-activated, becoming permeable by membrane potential changes that are under the minimal threshold to induce depolarization, and confer burst discharge and rhythmic firing properties to the cells that display them. T-type VGCCs do not appear to have auxiliary modulatory subunits, opposite to high-voltage-activated VGCCs as N-, R-, or P/Qtypes, and are expressed in the heart and central nervous system, where they are located in neurons of the brainstem, cerebellum, and cerebral cortex [9-11].

Mutations in CACNA1G, on chromosome 17q21.33, cause (1) childhood-onset psychomotor retardation with epilepsy, finger anomalies, and cerebellar atrophy in relation with a gain-of-function phenomenon, caused by impaired channel inactivation [12, 13]; (2) generalized absence epilepsy [14]; and (3) an adult-onset, dominantly inherited, progressive cerebellar ataxia designated SCA42 [15-18], in which the most frequent variant, c.5144G>A p. Arg1715His, involves the voltage-sensing segment 4 of domain IV, and causes a loss-offunction effect through a positive shift in the steady-state activation threshold [19, 20]. Mutations in $C A C N A 1 G$ have not been associated with vestibular dysfunction previously.

A heterozygous missense substitution in exon 38 of CACNA1G, c.6958G>T (p.Gly2320Cys), not previously registered in the ClinVar, PubMed Identifier or Human Gene Mutation Database, was found in the proband. It was similarly present in his symptomatic mother (case III-2) and absent in his unaffected brother (IV-1), therefore supporting this variant's causality.

Alpha-1G's canonical sequence comprises 2,377 aminoacids. Its position 2,320 is located on the C-terminus cytoplasmic loop, outside the channel's tetrameric structure. This terminal loop determines fast channel inactivation at its amino side and is implicated in anchoring the whole structure to the cell membrane at its carboxyl side, preventing or restricting its displacement [21-23]. The CACNA1G c.6958G>T (p.Gly2320Cys) variant is the closest one to the $\alpha 1 \mathrm{G} \mathrm{C}$-terminus reported to date, and it is possible that such a distal location may play a role in the episodic and benign nature of the described phenotype.

The main clinical manifestations in this kindred were episodic dizziness and unsteadiness of gait, variably accompanied by headache, cheek numbness, obtundation, and fatigue. The disorder was not progressive in nature, in spite of disease duration of 27 years in case III-2, and although various exploratory findings such as saccadic HIT responses, a positive Romberg sign, truncal ataxia, limb dysmetria, and impaired tandem gait were found on examination, the patients remained asymptomatic between episodes.

Vestibular function testing revealed saccadic bedside HIT responses and reduced VOR gains on v-HIT in both study cases, reflecting impairment of the visually enhanced VOR, which pointed at pathological involvement of vestibular, as well as of cerebellar structures [24]. Furthermore, worsening unsteadiness in darkness (in absence of proprioceptive sensory loss), an illusory sensation of movement triggered by head movements, and lack of symptoms in static conditions, constitute manifestations of vestibular dysfunction that were experienced

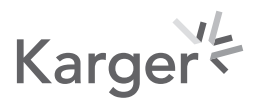


by both affected individuals. These episodic symptoms could be integrated in the "probable bilateral vestibulopathy" category [25], were it not for the simultaneous presence of transient cerebellar ataxia.

Farmer and Mustian [7] described a kindred from North Carolina with periodic attacks of vertigo and diplopia, designated periodic vestibulocerebellar ataxia, or EA4, which preceded a progressive ataxia that led to motor incapacity. Later reports of that same kinship, and of 2 other families related to it, included downbeat and gaze-evoked nystagmus, concomitant esotropia, altered ocular pursuit, progressive cerebellar ataxia and a lack of response to acetazolamide [26, 27]. With utmost respect for the work of those early authors and with the benefit of hindsight, as well as that of recent advances in the fields of episodic and progressive hereditary ataxias, it could be hypothesized that SCA6 or SCA38 could constitute possible alternative diagnoses for those kindreds because those diseases display downbeat nystagmus, vestibular dysfunction, diplopia and transient worsening of ataxia, while EA1, EA2, SCA1, 2, $3,4,5$, and dentatorubral-pallidoluysian atrophy, had been discarded by molecular studies $[28,29]$.

Our kindred were clinically distinct from EA3 due to lack of vertigo, tinnitus, and interictal myokimia [8] and a lack of response to acetazolamide, and also from EA4, as mentioned. Episodes were similar to those caused by EA2, although duration in the proband reached several months on severe occasions. Disease onset was during adulthood, as opposed to EA1 and other early onset EAs $[3,5]$. The presence of hemicranial headache and unilateral cheek numbness in the proband, led us to include migraine with aura in the differential diagnosis, and the fact that psychic and laboral stress-triggered attacks, made us consider a possible anxiety disorder.

Pharmacological intervention was necessary in case IV-2, due to the severity and prolonged duration of symptoms. Acetazolamide and topiramate worsened unsteadiness, and sumatriptan, amitriptyline or flunarizine did not relieve the headache. CBZ, however, proved consistently effective in bout prevention and provided symptomatic benefit in the proband, probably as a result of interaction of this drug with VGCCs [30]. The mildness of the episodic spells in case III-2 did not make necessary therapeutic measures.

Other genes involved in EA have also been linked to progressive ataxia, such as CACNA1A and $F G F 14$. The range of mutated $C A C N A 1 G$ phenotypes comprises progressive ataxia, epilepsy and developmental disturbances [12-14], while in this report, the occurrence of EA is added to that spectrum.

\section{Conclusion}

We communicate the clinical and molecular findings of a kindred presenting with a hitherto unreported episodic vestibulocerebellar ataxia, associated with a CACNA1G missense genetic variant. Further studies are needed to thoroughly investigate the clinical characteristics of this novel disease, which could be designated episodic vestibulocerebellar ataxia type 10 .

\section{Statement of Ethics}

In accordance with the World Medical Association Declaration of Helsinki and Hospital Universitario Miguel Servet institutional Ethics Committee, informed written consent was obtained for every test performed. Written informed consent was obtained from the patients or from their next of kin in case of decease, for publication of this case report and any accompanying videorecording. 


\section{Conflict of Interest Statement}

The authors have no conflicts of interest to declare.

\section{Funding Sources}

No funding was received for the elaboration of this Original Article.

\section{Author Contributions}

J. Gazulla: (1) conception AND design of the work; acquisition, analysis, and interpretation of data; (2) drafted the work and revised it critically for important intellectual content; approved the version to be published; and (3) agree to be accountable for all aspects of the work in ensuring that questions related to the accuracy or integrity of any part of the work are appropriately investigated and resolved. S. Izquierdo-Alvarez: (1) acquisition, analysis, and interpretation of data; (2) drafted part of the work and revised it critically for important intellectual content; approved the version to be published; (3) agree to be accountable for all aspects of the work in ensuring that questions related to the accuracy or integrity of any part of the work are appropriately investigated and resolved. E. Ruiz-Fernández: (1) acquisition and interpretation of data; (2) revised manuscript critically for important intellectual content; approved the version to be published; and (3) agree to be accountable for all aspects of the work in ensuring that questions related to the accuracy or integrity of any part of the work are appropriately investigated and resolved. A. Lázaro-Romero: (1) acquisition and interpretation of data; (2) revised manuscript critically for important intellectual content; approved the version to be published; and (3) agree to be accountable for all aspects of the work in ensuring that questions related to the accuracy or integrity of any part of the work are appropriately investigated and resolved. J. Berciano: (1) conception of work, and acquisition and analysis of data; (2) drafted part of the work and revised it critically for important intellectual content; approved the version to be published; and (3) agree to be accountable for all aspects of the work in ensuring that questions related to the accuracy or integrity of any part of the work are appropriately investigated and resolved.

\section{References}

1 Choi KD, Choi JH. Episodic ataxias: clinical and genetic features. J Mov Disord. 2016;9:129-35.

2 Riant F, Vahedi K, Tournier-Lasserve E. Ataxies épisodiques génétiques. Revue Neurologique. 2011;167(5): 401-7.

3 Conroy J, McGettigan P, Murphy R, Webb D, Murphy SM, McCoy B, et al. A novel locus for episodic ataxia: UBR4 the likely candidate. Eur J Hum Genet. 2014;22:505-10.

4 Piarroux J, Riant F, Humbertclaude V, Remerand G, Hadjadj J, Rejou F, et al. FGF14-related episodic ataxia: delineating the phenotype of episodic ataxia type 9. Ann Clin Transl Neurol. 2020;7:565-72.

5 Schwarz N, Bast T, Gaily E, Golla G, Gorman KM, Griffiths LR, et al. Clinical and genetic spectrum of SCN2Aassociated episodic ataxia. Eur J Paediatr Neurol. 2019;23:438-47.

6 Corbett MA, Bellows ST, Li M, Carroll R, Micallef S, Carvill G, et al. Dominant KCNA2 mutation causes episodic ataxia and pharmacoresponsive epilepsy. Neurology. 2016;87:1975-84.

7 Farmer TW, Mustian VM. Vestibulocerebellar ataxia. A newly defined hereditary syndrome with periodic manifestations. Arch Neurol. 1963;8:471-80.

8 Steckley JL, Ebers GC, Cader MZ, McLachlan RS. An autosomal dominant disorder with episodic ataxia, vertigo, and tinnitus. Neurology. 2001;57:1499-502.

9 Perez-Reyes E, Cribbs LL, Daud A, Lacerda AE, Barclay J, Williamson MP, et al. Molecular characterization of a neuronal low-voltage-activated T-type calcium channel. Nature. 1998;391:896-900. 
10 Benarroch E. Neuronal voltage-gated calcium channels. Brief overview of their function and clinical implications in neurology. Neurology. 2010;74:1310-5.

11 Huguenard JR. Low-voltage-activated (T-type) calcium-channel gene identified. Trends Neurosci. 1998;21: 451-2.

12 Chemin J, Siquier-Pernet K, Nicouleau M, Barcia G, Ahmad A, Medina-Cano D, et al. De novo mutation screening in childhood-onset cerebellar atrophy identifies gain-of-function mutations in the CACNA1G calcium channel gene. Brain. 2018;141:1998-2013.

13 Barresi S, Dentici ML, Manzoni F, Bellacchio E, Agolini E, Pizzi S, et al. Infantile-onset syndromic cerebellar ataxia and CACNA1G mutations. Pediatr Neurol. 2020;104:40-5.

14 Chen Y, Parker WD, Wang K. The role of T-type calcium channel genes in absence seizures. Front Neurol. 2014; 5:45.

15 Kimura M, Yabe I, Hama Y, Eguchi K, Ura S, Tsuzaka K, et al. SCA42 mutation analysis in a case series of Japanese patients with spinocerebellar ataxia. J Hum Genet. 2017;62:857-9.

16 Ngo K, Aker M, Petty LE, Chen J, Cavalcanti F, Nelson AB, et al. Expanding the global prevalence of spinocerebellar ataxia type 42. Neurol Genet. 2018;4:e232.

17 Li X, Zhou C, Cui L, Zhu L, Du H, Liu J, et al. A case of a novel CACNA1G mutation from a Chinese family with SCA42: a case report and literature review. Medicine. 2018;97:e12148.

18 Morino H, Matsuda Y, Muguruma K, Miyamoto R, Ohsawa R, Ohtake T, et al. A mutation in the low voltage-gated calcium channel CACNA1G alters the physiological properties of the channel, causing spinocerebellar ataxia. Mol Brain. 2015;8:89.

19 Coutelier M, Blesneac I, Monteil A, Monin ML, Ando K, Mundwiller E, et al. A recurrent mutation in CACNA1G alters Cav3.1 T-type calcium-channel conduction and causes autosomal-dominant cerebellar ataxia. Am J Hum Genet. 2015;97:726-37.

20 Hashiguchi S, Doi H, Kunii M, Nakamura Y, Shimuta M, Suzuki E, et al. Ataxic phenotype with altered CaV3.1 channel property in a mouse model for spinocerebellar ataxia 42. Neurobiol Dis. 2019;130:104516.

21 Staes M, Talavera K, Klugbauer N, Prenen J, Lacinova L, Droogmans G, et al. The amino side of the C-terminus determines fast inactivation of the T-type calcium channel alpha1G. J Physiol. 2001;530:35-45.

22 Mittman S, Guo J, Agnew WS. Structure and alternative splicing of the gene encoding alpha1G, a human brain T calcium channel alpha1 subunit. Neurosci Lett. 1999;274:143-6.

23 Uniprot.org [Internet]. CACNA1G voltage-dependent T-type calcim cannel subunit alpha-1G - Homo sapiens (Human) - CACNA1G gene \& protein. 2020. Available from: http://www.uniprot.org/uniprot/043497 Accessed 2020 May 15.

24 Migliaccio AA, Halmagyi GM, McGarvie LA, Cremer PD. Cerebellar ataxia with bilateral vestibulopathy: description of a syndrome and its characteristic clinical sign. Brain. 2004;127:280-93.

25 Strupp M, Kim JS, Murofushi T, Straumann D, Jen JC, Rosengren SM, et al. Bilateral vestibulopathy: diagnostic criteria consensus document of the Classification Committee of the Bàràny Society. J Vestib Res. 2017;27: 177-89.

26 Farris BK, Smith JL, Ayyar DR. Neuro-ophthalmologic findings in vestibulocerebellar ataxia. Arch Neurol. 1986;43:1050-3.

27 Small KW, Pollock SC, Vance JM, Stajich JM, Pericak-Vance M. Ocular motility in North Carolina autosomal dominant ataxia. J Neuroophthalmol. 1996;16:91-5.

28 Gazulla J, Orduna-Hospital E, Benavente I, Rodríguez-Valle A, Osorio-Caicedo P, Alvarez-de Andrés S, et al. Contributions to the study of spinocerebellar ataxia type 38 (SCA38). J Neurol. 2020;267:2288-95.

29 Damji KF, Allingham RR, Pollock SC, Small K, Lewis KE, Stajich JM, et al. Periodic vestibulocerebellar ataxia, an autosomal dominant ataxia with defective smooth pursuit, is genetically distinct from other autosomal dominant ataxias. Arch Neurol. 1996;53:338-44.

30 Sitges M, Chiu LM, Reed RC. Effects of levetiracetam, carbamazepine, phenytoin, valproate, lamotrigine, oxcarbazepine, topiramate, vinpocetine and sertraline on presynaptic hippocampal $\mathrm{Na}+$ and $\mathrm{Ca} 2+$ channels permeability. Neurochem Res. 2016;41(4):758-69. 